\title{
Blick auf die Grundsatzprogramme der Volksparteien - Eine gewerkschaftliche Position
}

Die Volksparteien werden sich immer ähnlicher, höhnen ihre Kritiker. Doch ist die Union wirklich nur so etwas wie eine „katholischere SPD“? Oder ist die CSU sozialer als die CDU, nur weil sie das Soziale im Firmenschild führt? Die Grundsatzprogrammarbeit liefert Aufschluss. Beurteilt durch eine „gewerkschaftliche Lesebrille“ steht die Frage im Mittelpunkt, was es für das gewerkschaftliche Wirken als Anwalt für Arbeit und Soziale Gerechtigkeit bedeuten kann, wenn praktische Politik sich künftig tatsächlich an den neu beschlossenen Grundsätzen ausrichtet. Was bedeutet es für die betriebliche, tarif- und gesellschaftspolitische Interessenvertretung der Gewerkschaften, vollzöge sich Regierungsarbeit entlang der jeweiligen Grundsatzorientierung?

\section{Was sagen Grundsatz- programme?}

Artikel 21 GG besagt: „Die Parteien wirken bei der politischen Willensbildung des Volkes mit“. Ihre Grundsatzprogramme sollten dem Volk Auskunft geben können, mit welchem normativen Anspruch Politik gestaltet wird, welchen Problemdeutungsmustern sich die Parteien verpflichtet fühlen, welche gesellschaftlichen Entwicklungsperspektiven ihnen als Leitbild dienen. Das verständliche Bemühen, populär und lesbar zu bleiben, erzwingt Konzessionen an Textlänge und Abstraktionsgrad. Mit dem Vorwurf, komplexe Probleme reduziert zu haben, sind Grundsatzprogramme daher schlecht zu kritisieren. Alle denkbaren Zukunftsherausforderungen können und wollen Grundsatzprogramme nicht abbilden. Der SPD geht es um „Soziale Demokratie im 21. Jahrhundert". ${ }^{1}$ Die CSU übertitelt: „Chancen für alle! In Freiheit und Verantwortung gemeinsam Zukunft gestalten". 2 Die CDU stellt ihr Grundsatzprogramm unter das Motto: „Freiheit und Sicherheit. Grundsätze für Deutschland“.3 So wird der Anspruch suggeriert, es ginge um mehr als nur um Grundsätze einer Partei. Selbst wenn es bloßes Marketing wäre, verglichen mit den anderen klingt hier eine Selbststilisierung als oberster Normgeber und Kursansager mit, wo bestenfalls christdemokratische Beiträge zum gesellschaftlichen Diskurs gemeint sein könnten. Parteimitglieder, -gänger sowie -widersacher, sie alle haben bei der Lektüre der Grundsatzprogramme ein höchst differierendes Leseinteresse. So gibt es auch eine „gewerk- schaftliche Lesart". Diese notiert weniger, was Zeitgeistanalytiker, Parteienforscher, Journalisten sowie Sprachästheten interessant finden mögen. Gewerkschaften beurteilen parteipolitische Positionierungen dieser Güte aus der Perspektive ihrer Arbeitsfelder. Wie wird auf Gewerkschaften Bezug genommen? Welche Aussagen zu gewerkschaftlichen Kernanliegen wie Tarifautonomie, Mitbestimmung, Vollbeschäftigung, Soziale Gerechtigkeit werden gemacht? Gibt es signifikante Interessenüberschneidungen oder Konflikte mit gewerkschaftlichen Problemwahrnehmungen und Lösungsperspektiven?

Die Parteitage haben letzte Worte sprechen können und nahezu einmütig die neuen Grundsatzprogramme autorisiert. Auf eine institutionalisierte Beratung bei der Programmarbeit durch Gewerkschaften legte nur die SPD Wert. Im gesamten Beratungszeitraum wurden immer wieder offizielle Gewerkschaftsmeinungen konsultiert. Der DGB-Vorsitzende konnte in der Programmkommission mitwirken. CDU und CSU begnügten sich mit Konsultation ihrer eigenen Arbeitnehmerorganisationen. CDU-Generalsekretär Ronald Pofalla hatte den DGB-Vorsitzenden im Mai 2007 wissen lassen, er werde sich ,sehr freuen, wenn der Deutsche Gewerkschaftsbund die anstehende Diskussion bereichern würde". Ein DGB-Schreiben vom Juni mit bereichernden Anregungen und Vorschlägen muss auf dem Postweg verloren gegangen sein. Die im November nachgereichte Kopie ist im beschlossenen Text ohne Resonanz geblieben.

\section{SPD - Fortschritt und Gerechtigkeit im 21. Jahrhundert}

\subsection{GRUNDWERTE}

Nach achtjähriger Diskussion und vierfachem Wechsel an der Parteispitze hat die SPD am 28.10.2007 ihr „Hamburger Programm" verabschiedet. Es ersetzt das Berliner Programm von 1989. Unverändert bleibt die Verankerung auf die Grundwerte Freiheit, Gerechtigkeit und Solidarität.

\footnotetext{
So das Leitmotto zum Vorwort des am 28. Oktober 2007 auf dem Hamburger Bundesparteitag beschlossenen Grundsatzprogramms. Es trägt den offiziellen Titel: „Hamburger Programm. Das Grundsatzprogramm der SPD" (http://www.parteitag. spd.de/servlet/PB/show/1731523/Hamburger\% 20Programm_final.pdf. Die Verweise und Zitate beruhen auf dieser Version mit 68 Textseiten).

2 Hier zitiert nach der 182 Textseiten umfassenden Version des Parteitagsbeschlusses der CSU vom 28. September 2007 (http://www.csu.de/home/Display/Politik/Grundsatzprogramm/Grundsatzprogramm).

3 „Freiheit und Sicherheit. Grundsätze für Deutschland" ", Beschluss des neuen Grundsatzprogramms auf dem 21. CDU-Parteitag am 03./04.12.07 in Hannover (http://www.grundsatzprogramm.cdu. de/doc/071203-beschluss-grundsatzprogramm-4. pdf. Verweise und Zitate beziehen sich auf die 369 durchnummerierten Abschnitte des 105-seitigen Textes).
}

Hans-Joachim Schabedoth, Dr., Bereichsleiter Gesellschaftspolitik/Grundsatzfragen beim Deutschen Gewerkschaftsbund. e-mail: achim.schabedoth@dgb.de 
Anders als die Union sieht die SPD keine Notwendigkeit, sie in eine Rangordnung zu zwingen. Die SPD versteht sich weiter „seit dem Godesberger Programm von 1959 als linke Volkspartei, die ihre Wurzeln im Judentum und Christentum, Humanismus und Aufklärung, marxistischer Gesellschaftsanalyse und den Erfahrungen der Arbeiterbewegung hat". Als linke Volkspartei bedankt sie sich für „wichtige Impulse der Frauenbewegung und (der) neuen sozialen Bewegungen "(Quelle: vgl. Fußnote 1, S. 13) Weder wohlmeinende noch übelwollende Kritik hielt die SPD davon ab, sich auf „die Idee des demokratischen Sozialismus" zu berufen (S.16f.). Ihre neue Wertefundierung liest sich zeitgemäßer, doch den eigentlichen Auftrag ihrer Grundsatzdebatte sah die Parteiführung nicht in der Suche nach neuen Grundwerteverankerungen, sondern in ihrer Anwendung auf neue Herausforderungen.

\subsection{GEWERKSCHAFTEN ALS AKTEUR}

Die Antworten des Hamburger Grundsatzprogramms berücksichtigen den zivilgesellschaftlichen Akteur Gewerkschaften nicht nur symbolisch. Mit zwölffacher Erwähnung wird den Gewerkschaften eine strategische Funktion für die Politik dieses Landes zugemessen. Sie werden genannt, wenn es um die Akteure geht, die nach dem Zweiten Weltkrieg große Fortschritte erstritten (S. 9). Es wird gelobt, Sozialdemokratie und Gewerkschaften hätten aus verachteten Proletarierinnen und Proletariern gleichberechtigte und selbstbewusste Staatsbürgerinnen und Staatsbürger gemacht (S. 12). Der Erfolg sozialdemokratischer Politik wird als abhängig gesehen vom demokratischen Engagement der Menschen, u. a. in den Gewerkschaften (S. 13). Die unverzichtbare Rolle des weltweiten Gewerkschaftsengagements wird anerkannt (S.23). Bei den Reflektionen zur solidarischen Bürgergesellschaft stehen die Gewerkschaften in einer Reihe mit den Parteien, Kirchen und Religionsgemeinschaften, Sozialund Umweltverbänden (S. 31). Die soziale Marktwirtschaft sieht das Hamburger Programm maßgeblich geprägt durch das Duo Sozialdemokratie und Gewerkschaften (S. 42). Starke Gewerkschaften gelten der SPD als unverzichtbar (S. 43). Es sei gemeinsame, zentrale Aufgabe von Unternehmen, Gewerkschaften und Politik, Fachkräfte und Qualifizierung zu fördern (S. 47). Mit den Gewerkschaften will die
SPD für einen gerechten Anteil der Arbeitnehmer am Ertrag der gesellschaftlichen Arbeit und für das Recht auf Mitbestimmung eintreten (S. 53). Und die SPD wünscht sich „starke und handlungsfähige Gewerkschaften, die große Teile der Belegschaften repräsentieren und streikfähig sind" (ebd.). Schließlich werden die vielen Menschen erwähnt, die sich für eine bessere und gerechtere Gesellschaft eben auch in den Gewerkschaften engagieren (S. 67).

\subsection{ARBEITSWELT UND SOZIALE GERECHTIGKEIT}

Ist diese gehäufte freundliche Bezugnahme auf die Gewerkschaften nur eine Kompensation für den rüden Umgangsstil in den letzten Jahren der Kanzlerschaft Gerhard Schröders (Schabedoth 2006)? Gegen eine solche Vermutung sprechen die weiteren Rekurse auf gewerkschaftliche Kernanliegen. Als hätte eine Aussage zur Bedeutung von Tarifautonomie nicht schon ausgereicht, bekräftigt die SPD ihre Wertschätzung für Tarifautonomie fünffach (S. 29, 43, 53, 55). Anerkannt wird: „Gute Arbeit schließt gesicherte Arbeitnehmerrechte ein. Die Mitbestimmung, die Betriebsverfassung, die Tarifautonomie, der Flächentarif, der Arbeits- und der Kündigungsschutz sind unverzichtbar" (S. 55). Das Thema Mitbestimmung, Kernanliegen der Gewerkschaften, wird nicht minder breit bedacht. Mitbestimmung soll auf europäischer Ebene gestärkt werden (S. 29, 43), sei Teil sozialer Demokratie (S. 19) sowie gemeinsame Grundlage für alle Sozialstaaten Europas (S. 28) und in der paritätischen Form in den Aufsichtsräten großer Unternehmen notwendig (S.43). Und schließlich sieht man Mitbestimmung als „Produktivkraft", die den sozialen Frieden gefördert hat (ebd.). Den Programmautoren ist offenkundig einsichtig, welchen zentralen Stellenwert Mitbestimmung für soziale Demokratie im 21. Jahrhundert besitzt (Hexel 2007). Wie nahe sich die Sozialdemokratie mit den Gewerkschaften über ihre Traditionswurzeln hinaus verbunden fühlt, zeigen weitere Programmaussagen an den Schnittstellen gemeinsamer Interessen und Arbeitsbereiche: Soziale Gerechtigkeit hat Leitfunktion (S. 5, 42, 68). Schon gebetsmühlenhaft beziehen sich die Sozialdemokraten bei 30 Gelegenheiten auf Teilhabeansprüche der Menschen. Globalisierung gilt ihnen nicht als Schicksalsmacht, sondern ist politisch gestaltbar. Die Verteilung von Einkommen und Vermögen müsse leistungsgerecht sein (S. 16). Der Staat habe vielfältigen Gestaltungsauftrag. Sozialdemokraten beharren auf dem „Primat demokratischer Politik und widersprechen der Unterwerfung des Politischen unter das Ökonomische" (S. 17). Internationale Institutionen und Organisationen sollen sich an Menschen- und Arbeitnehmerrechten messen lassen (S. 22). Bildung sei keine Ware, sondern ein Menschenrecht, dem zu dienen, der Staat verpflichtet sei (S.31). Aktivierender Staat und aktivierende Zivilgesellschaft werden nicht als Gegensatzpaar empfunden (S. 30f.). In der Arbeitswelt wird für die Gestaltung flexibler Arbeitszeiten und für mehr Zeitsouveränität plädiert (S. 41). „Märkte bedürfen der politischen Gestaltung - im Zeitalter der Globalisierung auch über nationale Grenzen hinaus" (S. 43). In einem eigenen Kapitel (S. 51ff.) wird definiert, was „Gute Arbeit für Alle“ bedeuten sollte. Passend zur tagespolitischen Konjunktur des Themas wird propagiert: "Wer Vollzeit arbeitet, soll mit dem Lohn auch seinen Lebensunterhalt bestreiten können. Wir kämpfen für existenzsichernde Mindestlöhne in Deutschland und Europa. Sie müssen tariflich und gesetzlich durchgesetzt werden“ (S. 54).

Sozialdemokratische Vollbeschäftigungspolitik gründet sich auf die vier Säulen: Förderung von qualitativem Wachstum und Innovation, koordinierte Arbeitsmarkt-, Bildungs-, Gleichstellungs- und Familienpolitik durch den vorsorgenden Sozialstaat, öffentlich geförderte und gemeinwohlorientierte Arbeit sowie auf eine moderne Arbeitszeitpolitik, „die Selbstbestimmung und Flexibilität fördert, sowie durch Arbeitszeitverkürzung mehr Menschen in Beschäftigung bringt" (S. 53). Mit dem "vorsorgenden Sozialstaat" hat die SPD ein neues Markenschild kreiert (Platzeck 2008). Erst nach intensiver Debatte in den Parteigliederungen und vielen Erläuterungen der Partei-Protagonisten (Beck/ Heil; Platzeck et al.; Albers/Nahles) sowie durch die Hamburger Parteitagsdebatte hat sich das anfängliche Missverständnis ausräumen lassen, es ginge um die Vernachlässigung nachsorgender Komponenten von Sozialstaatlichkeit. Der DGB-Vorsitzende Michael Sommer sieht im Hamburger Programm - über die traditionelle Nähe zwischen SPD und Gewerkschaften hinaus vielfältigste Ansatzpunkte für eine zukünftig verbesserte Zusammenarbeit (Sommer 2007, S. 47). Er gibt dabei zu bedenken: 
„Ein Parteiprogramm ist nie ein Gewerkschaftsprogramm und umgekehrt". Dem Hamburger Programm merkt man dies an, wenn auf die Tarifpolitik der Gewerkschaften rekurriert wird. So heißt es: "Wir wollen Lohnerhöhungen, die sich an Produktivität und Preissteigerungen orientieren " (S. 54). „Wir unterstützen Lohnzuwächse, die am Wachstum der Produktivität und an der Inflation orientiert sind" (S. 43). Und dreifach genäht hält besser:,Hohe Binnennachfrage schafft mehr Beschäftigung. Darum ist es nicht nur gerecht, sondern ökonomisch geboten, dass sich Lohnsteigerungen mindestens an der Produktivität und der Inflation orientieren" (S. 46). Jedem am gewerkschaftlichen wording geschulten Leser fällt auf, es fehlt die dritte Tarifkomponente: Umverteilung. Ist es $\mathrm{Ab}-$ sicht oder Unverständnis? Im Übrigen, warum sollte es nur „Lohnsteigerungen“ geben und nicht auch Gehaltssteigerungen? Gewerkschaften hätten hier allgemeiner von Einkommenssteigerungen gesprochen.

Eine Chance, sich vor den Konkurrenzparteien auszuzeichnen, haben auch die sozialdemokratischen Programmarbeiter ungenutzt gelassen. Zwar finden sich vielfältigste Hinweise zur Bedeutung öffentlicher Daseinsvorsorge. Doch einmal mehr unerwähnt bleibt die potenzielle Vorbildfunktion des öffentlichen Dienstes für alle Dimensionen "guter Arbeit“. Will der öffentliche Dienst - zumal bei Dienstherren mit SPD-Parteibuch - wirklich die Arbeitsverhältnisse nur so wie in der Privatwirtschaft üblich gestalten? Wie man weiß, hat der öffentliche Dienst durch eigene Arbeitszeitpolitik in jüngster Zeit sogar Schrittmacherdienste für allgemeine Rückschritte geleistet. In Abkehr von dieser Praxis könnte der öffentliche Dienst immerhin Prototyplieferant für solche Arbeitsbedingungen werden, die dazu beitragen, die allgemeinen Standards zu verbessern statt sie zu mindern.

\section{CDU - Freiheit und Sicherheit. Grundsätze für Deutschland}

\subsection{SCHATTENDASEIN FÜR GEWERKSCHAFTEN}

Die Christlich-Demokratische Union unterzog sich zum dritten Mal der Mühe, ein Grundsatzprogramm zu erarbeiten. Zum einen sieht man sich schon aus Wettbewerbsgründen angehalten, eine Sammlung eigener Problemdeutungen und Lösungsperspektiven zu erstellen. Zum anderen weiß man, dass sich seit ihrem 1994 beschlossenen Parteiprogramm die Welt massiv verändert hat. Nicht zuletzt gibt es die Erfahrung, kein Dauerabonnement auf Regierungsführung mehr zu haben. Die CDU muss von Wahl zu Wahl mehr Menschen situativ beeindrucken, die sich nicht länger mit Traditionsbotschaften des deutschen Konservativismus begnügen wollen. Im auffälligsten Kontrast zum sozialdemokratischen Grundsatzprogramm beansprucht die CDU erst gar nicht, in irgendeiner Weise gewerkschaftliche Erfahrungen zur Kenntnis nehmen zu wollen oder gar strategisch zu verarbeiten. Sie begnügt sich mit zwei eher lapidaren Bezugnahmen. Zum einen werden Gewerkschaften und die Arbeitgeberverbände aufgerufen, „Teile ihrer Verantwortung in die Betriebe abzugeben“ (Quelle: vgl. Fußnote 3, Abschnitt 173). Zum anderen geht es um das honorige Anliegen, bei der Integration von Zuwanderern mitzuwirken (309). Insgesamt bleibt die Rolle der Gewerkschaften als zivilgesellschaftlicher Akteur sowie als Betriebs- und Tarifpartei unterbelichtet bzw. weitgehend ausgeklammert. Die „Leistung der Tarifparteien“ "wird mit Bezug auf ihre Bringschuld für eine familienfreundliche Arbeitswelt erwähnt (82). In diesem Zusammenhang erscheint auch das Stichwort Tarifverträge. Sie sollten nach christdemokratischen Empfinden „für eine bessere Balance von Arbeit und Leben“" sorgen. Tarifverträge werden nochmals erwähnt, als etwas Starres, das „dezentral und nach spezifischen Bedürfnissen " ausgefüllt werden müsse (173). Unter der Überschrift „Arbeit für alle“ findet sich eine weitere Erwähnung der Tarifpartner. Es wird proklamiert: „Die soziale Marktwirtschaft will Vollbeschäftigung. Diesem Ziel sind Staat und Tarifpartner verpflichtet" (170). Inwieweit sich die CDU diesem Ziel verpflichtet fühlt, bleibt bei dieser Formulierung vage. Unter den Absätzen, die sich auf soziale Marktwirtschaft beziehen, wird „die Gewerbe- und Vertragsfreiheit, der Schutz vor Marktbarrieren und der Schutz vor Machtmissbrauch durch marktbeherrschende Unternehmen“ positiv benannt (143), doch kein Wort findet sich zur Koalitionsfreiheit. Sehr gelobt werden „Unternehmer und Unternehmensführer" (144f.). Gewerkschaften werden nicht einmal komplementär erwähnt. Man sollte trotzdem nicht vermuten, irgendein gewerkschaftsfeindlicher Zensor hätte - bis auf die zwei genannten - alle weiteren Stellen mit Bezugnahme auf Gewerkschaften getilgt. Viel naheliegender ist: Gewerkschaften als zivilgesellschaftliche Akteure besitzen in der politischen Wahrnehmung der meisten Christdemokraten keinen Stellenwert.

\section{DEFITIZE BEI ARBEITSWELTTHEMEN ...}

Wie die kurze Debatte um den Mindestlohn auf dem Parteitag zeigte, haben es Gewerkschafter mit christdemokratischer Wertorientierung offenbar immer noch schwer, mit eigenen Anregungen durchzudringen und damit die CDU auch programmatisch zu einer Volkspartei zu machen. „Mittelständische Unternehmen, Dienstleistungs- und Handwerksbetriebe sowie die Freien Berufe" firmieren als „Rückgrat unserer Wirtschaft“ (163), nicht aber die arbeitenden Menschen. Es fehlt Substanzielles zu guter Arbeit und gerechter Entlohnung. Viele Querverbindungen von allgemeinen Bekenntnissen zur Würde des Menschen und zur Situation in der Arbeitswelt sind den Autoren nicht geläufig. Dafür erscheinen die üblichen Dogmen aus dem Glaubensvorrat des Neoliberalismus: Staatsquote senken, Bürokratie abbauen, Arbeitsrecht weiter flexibilisieren, Bedingungen für Unternehmensfinanzierungen verbessern.

\section{... UND UNKLARE POSITIONEN}

Zum Arbeitsmarkt fällt den Programmautoren ein, es müsse „um einen fairen Ausgleich zwischen den legitimen Schutzbedürfnissen der Arbeitnehmer, den Beschäftigungschancen der Arbeitslosen und den Interessen der Unternehmen" gehen (172). Gewollt wird die „Flexibilisierung des Kündigungsschutzes“, dies wird sogar als „Gebot der Gerechtigkeit" behauptet. Es solle gelten: „Vorfahrt für Arbeit. In diesem Sinne muss die Aufgabe des fairen Interessenausgleichs gemeinsam mit den Tarifvertragsparteien angenommen werden" (ebd.). Wie man aus der tagespolitischen Praxis heraus vermuten darf, sind dabei eher Kollisionen als Koalitionen zu erwarten, wenn es bei der Weigerung bleibt, zwischen gefühlten und tatsächlichen Inflexibilitäten in der Arbeitwelt zu differenzieren 
(Pfarr et al. 2005). Weiter ist zu erfahren: „Soziale Partnerschaft, Tarifautonomie und Mitbestimmung in Form der Unternehmensmitbestimmung und der betrieblichen Mitbestimmung sind Grundlage unserer Wirtschafts- und Sozialordnung und Ausdruck der Sozialethik der christlichen Kirchen. Unser Verständnis von der Würde des arbeitenden Menschen verlangt seine Teilhabe an Entscheidungen, die die Bedingungen für seine Arbeitswelt setzen“. Und weiter wird präzisiert: „Die Mitbestimmung werden wir als Interessenvertretung der Arbeitnehmer zukunftsfähig, betriebsnah und beschäftigungsfreundlich fortentwickeln. Sie muss die zunehmende internationale Verflechtung der Unternehmen berücksichtigen“ (146).

Gewerkschafter lesen gerne in einem CDU-Programm: „Die Tarifautonomie ist ein Garant für die Stabilität des Standorts Deutschland“ (173). Beklagt werden aber zugleich „starre Branchentarifverträge“. Die CDU möchte ,zentral vereinbarte Rahmentarifverträge dezentral nach den spezifischen Bedürfnissen ausgefüllt" sehen. Diesen Prozess will sie „durch eine rechtssichere Gestaltung betrieblicher Bündnisse ergänzen". Es scheint nicht bewusst, dass dies im Konflikt steht zum vorangestellten Bekenntnis zur Tarifautonomie. Befürwortet wird die "Teilhabe der Arbeitnehmer" (auch der Arbeitnehmerinnen?) , am Erfolg und am Kapital der Unternehmen" (174). Jahresarbeitszeitkonten werden gesehen als Mittel, um „die Arbeit flexibler und produktiver“ zu organisieren (175). Und ,alle Bürger sollen Existenz sichernde Einkommen erzielen können“ (177). Dass es dabei nicht um eine Verpflichtung für Arbeitgeber geht, sondern die prinzipiell staatsskeptische CDU den Staat und seine Finanzmittel als Ersatz für solche Arbeitgeber heranziehen möchte, die existenzsichernde Löhne verweigern, das offenbart die Absicht, „niedrig entlohnte Arbeitnehmer finanziell unterstützen“ zu wollen. Doch Wettbewerb um die niedrigsten Löhne in Deutschland will die CDU auch nicht (178). „Sittenwidrige Löhne“, „die den ortsüblichen Branchenlohn deutlich unterschreiten", sollen verboten werden. Nach herkömmlichem Verständnis hieße dies jedoch, die CDU will Armutslöhne, die in zahlreichen Branchen schon ortsüblich sind, nicht bloß tolerieren, sondern bis zur Sittenwidrigkeitsgrenze (20-30\% weniger) dulden und sogar staatlich subventionieren.
Die CDU möchte „Arbeit für alle“, nennt aber keine plausiblen Wege zur Realisierung dieser Zielsetzung. Das Programm verbleibt vielfach bei der Wiederholung bekannter neoliberaler Rezepturen (Butterwegge et al. 2007). Die insgesamt in Deutschland unterentwickelte makroökonomische Debatte findet im CDU-Programm eine neue Abbildung. Allerdings gibt es mittlerweile in der CDU gewichtige Einzelstimmen, die es für eine „Lebenslüge" (Rüttgers 2007) halten, sich von immer weiteren Steuersenkungen verbesserte Beschäftigungschancen zu versprechen. Die Programmautoren haben sich von dieser Lebenslüge freilich nicht gelöst, sondern sie ins neue Grundsatzprogramm gerettet. Die steuerpolitischen Vorschläge erscheinen zumal unter dem Eindruck aktueller Erfahrungen mit Steuersenkungspolitik ganz erheblich unterkomplex. Was Positionierungen zur Sozialstaatsreformdebatte angeht, vermeiden die Programmautoren Kontinuitätsbrüche. Es finden sich lediglich semantische Zugeständnisse an die Kritiker der herkömmlichen CDU-Placebos. So heißt die Kopfpauschale in der Krankheits- und Pflegeversicherung nicht mehr „Kopfpauschale“, sondern es wird von ,solidarischen Prämienelementen “ gesprochen $(188,197)$.

Erstaunlich naiv wird auch das Thema „Verlagerung von Arbeitsplätzen ins Ausland" behandelt. Dazu postulieren Christdemokraten: „Wir erwarten aber von den Unternehmern, dass sie solche Entscheidungen verantwortungsvoll unter der Berücksichtung der sozialen und gesellschaftlichen Folgen treffen und sich für den Standort Deutschland einsetzen" (148). Was aber ist, wenn Unternehmer nicht so verantwortungsvoll sind, wie das die CDU erwartet? Die Aussagen zum „Schutz der Freiheit der internationalen Märkte" bleiben widersprüchlich. Zwar plädiert man für „eine international abgestimmte Wettbewerbsordnung“ (154), gleichwohl: „im Zweifelsfalle hat jedoch die Freiheit des Marktes Vorrang" (155). Weniger konkret sind Programmaussagen, die sich direkt oder indirekt auf die internationalen Finanzmärkte beziehen. Sie nähren den Eindruck, es wirkten unveränderbare Schicksalszwänge. Wenn es um unternehmerische Entscheidungen und ökonomische Kalküle geht, verstummt der christdemokratische Gestaltungsanspruch oder zieht sich auf Appelle an die Einsichtsfähigkeit der Unternehmer zurück. Da dieses Argumenta- tionsvakuum an keiner anderen Stelle gefüllt wird, liegt der Schluss nahe, im CDUProgrammvorrat soll es keinen Primat des Politischen vor dem Ökonomischen geben. Das erklärt die anti-etatistischen Einsprengsel an vielen Programmstellen. Diese fehlen signifikant gerade dort, wo es um staatliche Subventionen für Armutslöhne geht. Der weitere politische Diskurs wird klären müssen, inwieweit Deutschland wirklich akzeptiert, was die CDU hier zu „Grundsätzen für Deutschland“ erklärt.

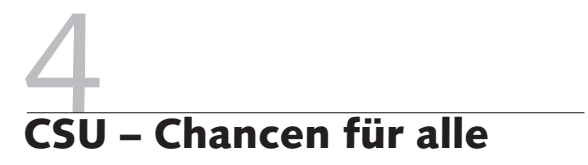

\subsection{EIN NEUES PROGRAMM WIDER WILLEN}

Der christsoziale Unionsteil eilte der Schwester mit seiner Beschlussfassung zum Grundsatzprogramm am 28./29. September 2007 zeitlich voraus. Es ist das sechste Grundsatzprogramm, mit dem die Christlich-Sozialen „den Veränderungen und den Herausforderungen zu Beginn des 21. Jahrhunderts gerecht werden" wollen (Quelle: vgl. Fußnote 2, S. 9).

Für die CSU erläutert Alois Glück, der Vorsitzende der Grundsatzkommission seiner Partei, eigentlich gäbe es gar keine Notwendigkeit, „die grundsätzlichen Positionen des bisherigen Grundsatzprogramms, beschlossen 1993“, zu verändern, sie seien weiterhin zutreffend für das Selbstverständnis der CSU. Er sieht die neuen Debatten motiviert durch das enttäuschende Abschneiden bei der Bundestagwahl. Man erinnert sich: Die Unionsparteien waren sich sehr sicher, mit ihrem neoliberal eingefärbten Wahlprogramm eine Regierungsmehrheit mit der FDP gewinnen zu können. Es ist bekanntlich anders gekommen. Und im „Merkelland“ (Meng 2005) schien es zum Missbehagen der CSU nicht mehr opportun, Ursachenforschung zu betreiben. Insofern haben die Grundsatzdebatten der Union auch die Nebenfunktion, über die politische Verortung neu nachzudenken. „Der Prozess der Neufassung wird auch ein Prozess der Klärung und Selbstvergewisserung über Identität und Kursbestimmung unter den Bedingungen des politischen Handelns in dieser Zeit sein" (Glück 2006, S. 10).

Im Vergleich mit den Angeboten von SPD und CDU kommt der CSU-Beitrag 
mit seinen 175 Textseiten ziemlich weitschweifig daher. Die CSU muss ein Sonderproblem bearbeiten. Sie ist erklärtermaßen die (!) Bayern-Partei, will aber in ihrem Grundsatzprogramm Aussagen treffen, die sich auf Deutschland, Europa und die Welt beziehen. Als Nicht-Bundespartei ist ihr offensichtlich klar, wie sehr ihre regionale Verankerung Basis bundespolitischer Relevanz ist. Insofern finden sich in einer Breite, die es bei CDU und SPD nicht gibt, Bekenntnisse zu Brauchtum und Heimat. $\mathrm{Zu}$ den Gewerkschaften sind der CSU nur zwei Bezüge wichtig, zu den Heimatvertriebenen drei und zum allgemeinen Thema Heimat sogar 42, was sich andere Parteien eher für kommunalpolitische Programme reservieren würden. Man sollte das trotzdem nicht als provinziell abstempeln. Nach ihrem Selbstverständnis ist die CSU gerade deshalb weltoffen und gegenüber anderen Kulturen austauschfähig, weil sie einen profilierten eigenen Wertebestand hat, dessen Respekt sie von allen fordert, die im Gegenzug dann aber auch Respekt und Toleranz erwarten können. Aus der gewerkschaftlichen Perspektive mögen der gehäufte Umgang der CSU mit Heimatverbundenheit, christlichen Grundwerten und das häufige Paraphrasieren des Subsidiaritätsprinzips ermüdend oder uninteressant wirken. Wer nach der politischen Substanz des Programms sucht, muss durch dieses Platitüdendickicht hindurch.

\subsection{ARBEITSWELT:} TERRA INCOGNITA...

Wie ihrer großen Schwester bleibt der CSU die Arbeitswelt terra incognita. Gemessen an ihren Wahlerfolgen ist die CSU sogar mehr Volkspartei als die CDU. Wo die große Schwester sich mit übergroßer Wertschätzung für die Unternehmer vortut, weiß die CSU immerhin: „Die Arbeitnehmer sind das Rückgrat von Wirtschaft und Gesellschaft. Ihr Einsatz und ihre Schaffenskraft sind eine der Grundlagen für den wirtschaftlichen und sozialen Wohlstand in Bayern und Deutschland“ (S. 64). So „bekennt" man sich auch zur Tarifautonomie, Mitbestimmung und Sozialpartnerschaft. „Mitbestimmung und Mitbeteiligung der Arbeitnehmer haben sich in unserer Arbeitswelt bewährt. Die Arbeitnehmerorganisationen haben für die gesellschaftliche und wirtschaftliche Entwicklung einen wesentlichen Beitrag geleistet“.

\section{...UND WIDERSPRÜCHLICHES}

Doch bleiben Zweifel, ob den Programmautoren die Konsequenzen ihres Bekenntnisses zur Tarifautonomie bewusst sind. Seitenlang und wiederholt hat die CSU die Leser ihres Grundsatzprogramms damit vertraut gemacht, nach dem Subsidiaritätsprinzip sei die Selbstorganisation der Betroffenen zweckgemäßer als staatliches Besserwollen. Umso erstaunlicher ist die einzige Ausnahme im Programm. Dabei geht es um das Agieren der Sozialpartner: „Wir wollen die bewährte Sozialpartnerschaft auf der Grundlage der sozialen Marktwirtschaft weiter entwickeln und für die wissensbasierte Industrie- und Dienstleistungsgesellschaft zukunftsfest machen". Und: „Die Tarifparteien halten mit den Entscheidungen über Tarifabschlüsse einen wichtigen Schlüssel für die Zukunft der Arbeit in der Hand. Unter Wahrung der Tarifautonomie wollen wir die notwendige Flexibilität und kalkulierbare Sicherheit für beide Seiten - für Arbeitnehmer und Arbeitgeber" (S. 65). Doch warum will die CSU das nicht den vorab so gelobten Sozialpartnern oder Tarifparteien alleine überlassen? Wieso „wir“? Die CSU in ihrer Eigenschaft als Arbeitgeber der hauptamtlich Beschäftigten ihrer Partei wird wohl nicht gemeint sein. Also die CSU als Partei und Mitinhaber von Regierungsmacht? Auf allen anderen Feldern der Gesellschaftspolitik werden die jeweils Zuständigen auf den unteren Ebenen für regelungskompetent gehalten. Den Tarifvertragsparteien spricht man es offenbar ab und dient ihnen - wie auch von Seiten der CDU - betriebliche Bündnisse für Arbeit an. Da das Aushandeln von Löhnen und Gehältern ausdrücklich den Tarifvertragsparteien überlassen bleiben soll, leuchtet die Sinnhaftigkeit dieser Bevormundung nicht ein. Was mag gemeint sein, wenn die CSU meint: „Wir wollen Einstellungshürden beseitigen und Mitarbeitern und Unternehmen mehr Vertragssouveränität geben" (ebd.). Geschickter als die CDU versteht es die CSU sich auf diese Weise Hintertüren für den Abbau von Kündigungsschutzrechten offen zu halten. Trotz jahrzehntelanger Debatten über gesellschaftliche Verteilungsfragen findet sich die törichte Behauptung von der „Spaltung der Gesellschaft in Arbeitsplatzbesitzer und Arbeitslose" (ebd.). Zwar ist die empirisch signifikantere Spaltungslinie zwischen Arbeitsplatzanbietern und Arbeitsplatzsuchenden damit nicht geleugnet, doch es mutet immer wieder verwunderlich an, die derzeit Beschäftigten für „Arbeitsplatzbesitzer“ $\mathrm{zu}$ halten.

\subsection{THEMA INTERESSENVERTRETUNG: EINE LEERSTELLE}

Mit dem vormundschaftlichen Besserwissen über den Sinn „betrieblicher Bündnisse für Arbeit" kontrastieren die Leerstellen, wo eigentlich Parteien, die sich in der Wirklichkeit unseres Landes verankert fühlen, die Rolle des zivilgesellschaftlichen Akteurs Gewerkschaften strategisch verarbeiten müssten. Nur ihre Rolle in der Tarifpolitik wird vage beleuchtet. Die CSU gönnt den Gewerkschaften darüber hinaus eine zweite marginale Erwähnung: „Für die CSU ist das soziale Leben in Familien, Nachbarschaften, privaten Initiativen, Vereinen, Kirchen und Gewerkschaften besonders wertvoll“" (S. 57). Konsequenzen daraus werden nicht gezogen. Wie schon die CDU hat die CSU die Gewerkschaften einfach nicht „auf ihrem Film“. Es ist erstaunlich, wie wenig sich die Volkspartei CSU darum bemüht, auf Wahrnehmungen ihrer tatsächlichen und potenziellen Wählerinnen und Wähler mit gewerkschaftlicher Erfahrung und Bindung einzugehen. Das Programm legt ausführlichst dar, wie man in Freiheit und Verantwortung „gemeinsam Zukunft gestalten" möchte, findet aber kein einziges Wort zum Wirken von Betriebs- und Personalräten. Nichts fällt der CSU ein zum System institutionalisierter Machtkontrolle über Mitbestimmung. Muss der Staat wirklich der „dumme August “bleiben, wenn die so wortreich gelobten Unternehmer sich nicht so einsichtsvoll verhalten, wenn es um Entscheidungen zur Berufsausbildung, Weiterbildung, Vereinbarkeit von Familie und Beruf, um bessere Kundenbezüge, neue Märkte und neue Produkte geht? Gelobt werden „,engagierte Unternehmer, mutige Wirtschaftspioniere und verantwortungsvolle Führungskräfte“ (S. 67). Aber was ist mit den nicht engagierten, mit den phantasielosen und verantwortungsscheuen unternehmerischen Kräften? Bayerische Staatskanzlei und Siemens-Konzernleitung liegen nicht so weit auseinander, da hätte man durchaus eine Menge Fakten zur Kenntnis nehmen können, die zum Relativieren pauschaler Lobeslieder auf „engagierte Unternehmer, mutige Wirtschaftspioniere und verantwortungsvolle Führungskräfte" nötigen. 
Die Zukunft der Arbeit hängt nicht nur davon ab, dass die Arbeitnehmer als „Rückgrat von Wirtschaft und Gesellschaft" und die Unternehmer mit sozialer Verantwortung ihre Pflichten erfüllen. Der CSU ist das immerhin bewusst, wenn sie über Bayern und Deutschland hinausschaut: „Fairer Wettbewerb braucht Regeln, Freihandel und wirtschaftliche Interessen dürfen nicht dazu führen, über Verletzungen der Menschenrechte und den Raubbau an der Schöpfung hinweg zu sehen. Einsatz für die Menschenrechte, Schutz der Umwelt, des Klimas und der Weltmeere, Entwicklungszusammenarbeit und internationale Sicherheit - das alles sind keine regionalen Aufgaben, keine Aufgaben verschiedener Welten. Das sind die großen Aufgaben unserer gemeinsamen Welt" (S. 21). Wer das in den internationalen Zusammenhängen erkannt hat, dem hätte man durchaus auch die Einsicht gewünscht, im bayerischen und nationalen Rahmen intelligenter zwischen notwendiger Regulierung und Bürokratielasten sowie zwischen staatlicher Bevormundung und unverzichtbarer Rahmensetzung differenzieren zu können. Die proklamierte „moderne wertorientierte Volkspartei“ (S. 177) kann die CSU umso eher werden, wie sie sich wirklich mit dem auseinandersetzt, was Lebens- und Arbeitsprobleme der Menschen sind. „Die CSU hält am Ziel der Vollbeschäftigung fest" (S. 62). Aber was bedeutet das für die Verantwortung des Staates als Arbeitgeber, als Mitgestalter von Arbeitsmarktpolitik, als Grenzensetzer unternehmerischen Handelns, als makroökonomischer Akteur? Das CSU-Grundsatzprogramm schweigt sich aus oder flüchtet in Radio-Eriwan-Botschaften.

\section{Fazit}

Die Verteilung markanter Catchwords in den drei Parteidokumenten darf sicher nicht politisch überinterpretiert werden. Doch bleibt es eine signifikante Aussage, mit welcher Intensität sich die Parteien auf Gewerkschaften und auf Sachverhalte beziehen, die für ihr Arbeiten eine zentrale Bedeutung haben (Tabelle 1).

Geht es um die Bezugnahme auf Gewerkschaften, haben die Sozialdemokraten ein Alleinstellungsmerkmal. Auch zum Themenfeld Tarifpolitik hat das SPDGrundsatzprogramm eine klare Sicht, die

\begin{tabular}{lccc|}
\hline \multicolumn{4}{l}{ Tabelle 1: Ausgewählte Catchwords in den Grundsatzprogrammen } \\
\hline Partei & SPD & CDU & CSU \\
Catchwords & & 2 & 2 \\
Gewerkschaften & 11 & 2 & 2 \\
Tarifautonomie & 5 & 3 & 3 \\
Mitbestimmung & 12 & 21 & 9 \\
Teilhabe (teilhaben) & 30 & 3 & 0 \\
Soziale Gerechtigkeit & 5 & 8 & 42 \\
Heimat & 4 & & WSI MITTEILUNGEN \\
Quelle: Zusammenstellung des Autors. & & &
\end{tabular}

nicht durch Relativierungen wie bei den Unions-Schwestern wieder infrage gestellt wird. Mitbestimmung und Teilhabe sind Fundamente sozialdemokratischer Politik, während CDU und CSU es bei affirmativer Erwähnung belassen. Die Vokabel „Soziale Gerechtigkeit" sucht man bei den Christsozialen vergeblich. Dafür sind sie unübertrefflich, wenn es um Heimatliches geht.

Die CDU zeigt sich bemüht, den maßgeblich von der Kanzlerin Angela Merkel bestimmten Kurs vorsichtiger Modernisierung auch programmatisch nachzuholen. Dazu geht es um einen aktuellen Katalog zu zentralen Dimensionen christdemokratischer Problemwahrnehmung und -verarbeitung. Dem aktuellen Grundsatzprogramm ist eine missdeutungsfreie Normsetzung für zukünftiges politisches Handeln wohl weniger wichtig als das Bemühen, die Programmatik auf die Höhe der Zeit zu bringen, ohne Optionen für alle möglichen Koalitionen und Neuakzentuierungen zu verbauen. Die Sozialdemokraten haben sich diesbezüglich mehr Präzision verordnet als sich die Unionsparteien erlauben wollten oder konnten. Sicherlich haben CDU und CSU somit ihr Ziel erreicht, eine aktuelle Zusammenstellung zur Bandbreite parteiinterner Debattenlagen zu erstellen. Missglückt ist jedoch der Versuch, den Lesern in Abgrenzung zum sozialdemokratischen Konkurrenten einen originären und konsistenten Gesellschaftsentwurf zu präsentieren. Wollte man den eigenen Mitgliedern und Anhängern bloß demonstrieren, dass man die modisch gewordenen Versatzstücke aus den Debatten der letzten Jahre in ein aktuelles Grundsatzprogramm zu integrieren weiß, dann ist das fraglos gelungen. Bei der CDU merkt man es den Positionen zur Familienpolitik und zur Ökologie mehr an als bei den Arbeitsweltthemen und bei der Ökonomie. Neue Erfahrungen, kollektive Lernfortschritte einer ganzen Nation sowie heute besser erkenn- bare Zukunftsherausforderungen finden in den drei Programmen sehr ungleichen Niederschlag. Leser, die sich für Parteiprogrammatik nicht nur beruflich oder aus verbandsstrategischen Erwägungen heraus interessieren, dürfte überraschen, wie kritiklos das Abfeiern von Reform-Placebos und der hinreichend verdächtigen marktradikalen Problemantworten nunmehr Eingang in die Grundsatzprogramme der christdemokratischen Volksparteien gefunden hat. Arbeitsweltthemen standen bei ihnen nicht im Fokus. Identitätsprägend bleibt der Stellenwert moderner Familienpolitik, und gelungen ist das Aufpolieren neoliberaler Grundüberzeugungen, die im Regierungsalltag an Strahlkraft eingebüßt haben.

Was politischen Programmatikern vom Kaliber eines Erhard Eppler, eines Heiner Geissler oder eines Hans Maier wohl noch als Verstoß gegen intellektuelle Redlichkeit galt, ist Programm-Machern von heute erlaubte List, um heterogenen Erwartungen gerecht $\mathrm{zu}$ werden. Programmaussagen müssen offenbar nicht mehr möglichst widerspruchsfrei sein. CDU und CSU sind zum Beispiel beides: die Befürworter eines starken Staates als Ordnungsmacht und zugleich die Befreier des Einzelnen, wenn es darum geht, sie vor staatlicher Bevormundung durch soziale Leistungen oder Rechte in der Arbeitswelt zu schützen. Beide Unionsschwestern bekennen sich zur Tarifautonomie, kokettieren aber mit betrieblichen Bündnissen, um sie zu unterminieren. Wenn das nicht widersprüchlich ist, ist es doppelzüngig. Gilt nicht gerade für Parteien auf christlichem Wertefundament: „Eure Rede aber sei, ja, ja; nein, nein. Was darüber ist, das ist von übel" (Matthäus 5,37$)$. Im weiteren politischen Diskurs, der durch die Grundsatzprogramme befördert werden soll, darf man den Parteien solche Doppelbödigkeiten nicht durchgehen lassen. Die Bürgerinnen und Bürger wären gut gewappnet, die Debatte 
um konkretes Alltagshandeln der Politik mit der Messlatte des Grundgesetzes zu beurteilen. Zu denken ist dabei an den Artikel 1, Menschenwürde, Grundrechtsbindung der staatlichen Gewalt, an Artikel 9, Vereinigungs-, Koalitionsfreiheit sowie an Artikel 20, Sozialstaatsprinzip. Die Vorliebe für Sowohl-als-auch-Aussagen schränken den Wert von Grundsatzprogrammen als politische Kursanzeiger nicht unwesentlich ein. Parteien mit christlicher Fundierung werden wissen: Letztendlich zählen die Taten und nicht (gedruckte) Worte. Die SPD immerhin hat die Dissonanz zwischen den heterogenen Erwartungen ihrer Mitglieder und Anhänger an sozialdemokratische Politik und der Alltagsarbeit in der Regierungsverantwortung mit dem neuen
Grundsatzprogramm ein gutes Stück aufgelöst (Eppler 2007). Auch CDU-Generalsekretär Ronald Pofalla gibt sich überzeugt, die eigene Grundsatzprogrammarbeit habe sich gelohnt:

„Ein Grundsatzprogramm ist in der Tat kein Text, den man wöchentlich oder noch öfter liest. Aber wir brauchen einen inhaltlichen Kompass, ein klares Fundament. Wer sich für die CDU interessiert, wer uns wählt, muss wissen, wofür wir stehen, was unsere Ziele und Überzeugungen sind. Wir beschreiben mit dem Grundsatzprogramm deshalb, wie wir uns als Christliche Demokraten verstehen und welche Werte uns leiten. Das liest man nicht jeden Tag beim Frühstück, aber es bestimmt, was man nach dem Frühstück macht“ (Pofalla 2007, S. 11).
Den Vorrang der täglichen Arbeit vor dem gedruckten Wort proklamiert die CSU sogar im Vorwort: „Zugleich wissen wir, dass uns nicht in erster Linie theoretische Programme verbinden, sondern unsere tägliche Arbeit für die Menschen“" (S. 97). Mit welcher Kursrichtung, welchen noch erlaubten oder grundsatzwidrigen Abweichungen sich diese tägliche Arbeit vollzieht, dafür bieten alle drei Grundsatzprogramme eine aktuelle Messlatte. Für die Interessenvertretungsarbeit der Gewerkschaften ist es keineswegs unerheblich, ob die einen oder die anderen eine parlamentarische Mehrheit gewinnen und damit ihren Grundsätzen folgen könnten. Mindestens das verrät auch das geduldigste (Grundsatzprogramm-)Papier.

\section{LITERATUR}

Albers, D./Nahles, A. (Hrsg.) (2007): Linke Programmbausteine. Denkanstöße zum Hamburger Programm der SPD, Berlin

Beck, K./Heil, H. (Hrsg.) (2007): Soziale Demokratie im 21. Jahrhundert, Berlin

Butterwegge, C./Lösch, B./ Btak, R. (2007): Kritik des Neoliberalismus, Wiesbaden

Eppler, E. (2007): Die Zeit ist reif, Gespräch mit Erhard Eppler, in: Neue Gesellschaft/Frankfurter Hefte 11, S. 32-38

Glück, A. (2006): Kompass und Kompetenz, in: Die politische Meinung 437, S. 9-13

Hexel, D. (2007): Zum Standort der Unternehmensmitbestimmung heute, in: Ständige Deputation des deutschen Juristentages (Hrsg.): Verhandlungen des sechsundsechzigsten Deutschen Juristentages, München, S. $49-75$

Horn, G. A. (2007): Grundlagen und Kritik der neoliberalen Wirtschaftspolitik, in: WISO Wirtschafts- und sozialpolitische Zeitschrift des Instituts für Sozial- und Wirtschaftswissenschaften 1, S. 19-48

Meng, R. (2006): Merkelland, Köln
Pfarr. H./Ullmann, K./Bradtke, M./Schneider, J./Kimmich, M./Bothfeld, S. (2005): Der Kündigungsschutz zwischen Wahrnehmung und Wirklichkeit, München und Mehring

Platzeck, M. (2008): Der vorsorgende Sozialstaat, in: Sommer, M./ Schabedoth, H.-J., Europa sozial gestalten!, S. 64-70

Platzeck, M./Steinmeier, F.-W./Steinbrück, P. (Hrsg.) (2007): Auf der Höhe der Zeit. Soziale Demokratie und Fortschritte im 21. Jahrhundert, Berlin

Pofalla, R. (2007): „Wir wollen die Chancengesellschaft", Ronald Pofalla im UNION-Gespräch, in: UNION 2, S. 10-15

Rüttgers, J. (2007): Die Marktwirtschaft muss sozial bleiben. Eine Streitschrift, Köln

Schabedoth, H.-J. (2006): Unsere Jahre mit Gerhard Schröder, RotGrüne-Regierungsarbeit zwischen Aufbruch und Abbruch, Marburg Sommer, M. (2007): „Ein Schritt der Normalisierung “, Gespräch mit Michael Sommer, in: Neue Gesellschaft/Frankfurter Hefte 11, S. 45-48 